\title{
Dietary reference intake (DRI) value for dietary polyphenols: are we heading in the right direction?
}

\author{
Gary Williamson ${ }^{1 *}$ and Birgit Holst ${ }^{2}$ \\ ${ }^{1}$ Chair of Functional Food, Procter Department of Food Science, University of Leeds, Leeds LS2 9JT, UK \\ ${ }^{2}$ Nestlé Waters M T, PTC Water, BP 101, 88804 Vittel CEDEX, France
}

Dietary Reference Intake (DRI) values exist for vitamins and minerals, and provide a guideline on the optimal dose range to avoid deficiency and prevent toxicity. Polyphenols are widely distributed in plant foods, and have been linked to improved human health through reduced risk of chronic diseases, especially cardiovascular. Although they do not cause classical deficiencies, recently they have been discussed as 'lifespan essentials because they are needed to achieve a full lifespan by reducing the risk of a range of chronic diseases. A recent meta analysis shows promising actions of polyphenols from cocoa, soya and tea on flow mediated dilation, blood pressure and LDL cholesterol. Many epidemiological studies support the action of polyphenols or polyphenol-rich foods on health, but there are still many gaps in our knowledge. More adequately powered, randomised, placebo controlled human studies are needed on polyphenols. There is a large number of structurally different polyphenols which are relevant for health, and obtaining enough information to set a DRI for each of these will not be feasible in the foreseeable future. A new approach is needed, and a new way of thinking, which would apply not only to polyphenols but also to other phytochemicals. Today, a target intake value of polyphenols as 'lifespan essentials' needs to be based on the amount of polyphenols in '5-a-day'. We are heading in the right direction towards a DRI, but bioavailability and dose-effects, including toxic levels, need to be established before DRIs can be considered.

Polyphenols: Bioavailability: Chronic disease prevention: Lifespan essential: Dietary requirements

This is not intended to be a comprehensive review of the literature as several in-depth reviews have appeared in the last few years which summarize the results of human studies in relation to polyphenols ${ }^{(1-9)}$. Furthermore, although isoflavones are polyphenols, we have not made recommendations for this class.

\section{What are polyphenols?}

Significant research effort is now focused on minor dietary constituents, vitamins and trace elements, phytochemicals (carotenoids, flavonoids, indoles, isothiocyanates, etc.), zoochemicals (conjugated linoleic and $n-3$ fatty acids, etc.), fungochemicals and bacteriochemicals (formed during food fermentations and by the gut microflora).

The best studied polyphenols (also called flavonoids, and belonging to the bigger class of phytochemicals or phytonutrients) are (epi)catechins (including green tea polyphenols) and procyanidins (class: flavanols), quercetin (class: flavonols), and the isoflavones (genistein and daidzein, found in soya). Other, less studied classes, at least for health effects in humans, are anthocyanins and hydroxycinnamic acids.

\section{Biological activities}

All polyphenols share several structural features, particularly phenolic rings, and are amongst the most powerful of all the dietary antioxidants in vitro. This property led to extensive use of the word 'antioxidant', and overall polyphenol content of a food source is commonly evaluated by measuring the antioxidant activity e.g. by TEAC, ORAC, TRAP, etc ${ }^{(10)}$. In the past, direct conclusions from in vitro antioxidant activity of a food were extrapolated to health benefits in vivo in humans. However, it is now clear that this was far too simplistic. Polyphenol action in vivo is through indirect antioxidant protection by activating endogenous defence systems, modulating cellular signalling processes involving nuclear factor-kappa B (NF- $\kappa \mathrm{B})$, activator protein-1(AP-1) DNA binding, glutathione biosynthesis, phosphoinositide 3 (PI3)-kinase/ protein kinase $\mathrm{B}$ (Akt), mitogen-activated protein kinase (MAPK), translocation into the nucleus of nuclear factor erythroid 2 related factor 2 (Nrf2), and inhibition of oxidative or pro-inflammatory enzymes such as cyclo-oxygenase, lipoxygenase, NADPH oxidase, and some metalloproteins. For example, the inhibition of one specific enzyme, COX-2, affects inflammation, and consequently the development of a larger number of chronic diseases ${ }^{(11)}$. Epicatechin, a polyphenol found in cocoa, green tea and many fruits, affects nitric oxide formation via NADPH oxidase ${ }^{(12,13)}$, hence modifying vascular biomarkers such as flow mediated dilation, an important diagnostic marker of cardiovascular risk ${ }^{(14)}$.

Based on the current state of the art, and especially on published clinical studies, flavanols ((epi)catechins in fruits, procyanidins in fruit and cocoa, galloylated catechins in tea), and the flavonols (mainly quercetin, in vegetables, tea and some fruits) are believed to be the most important 
polyphenols for human health; actions of the other classes (flavanones, hydroxycinnamates and anthocyanins) are less studied in human intervention trials. However, acceptance of health effects by the nutrition community is mixed, since the effects are subtle and these types of dietary compounds do not meet the classical criteria of being essential nutrients for growth and development.

Most polyphenols are absorbed but the extent of absorption and metabolism depends on the class of polyphenol. Ileostomy, human in situ perfusion and intervention studies on healthy subjects show high absorption of some classes. Human studies show a poor absorption of intact anthocyanins and procyanidins ${ }^{(5)}$. However, for the latter, it remains to be seen if the microbial metabolites play an important role in biological functions. Bioavailability from different matrixes needs to be established, since the route and extent of absorption are as important as the extent and pathway of metabolism, which may lead to an inactivation and fast excretion of some polyphenols. For others, metabolites (small intestinal, liver or gut microbial), may have an equal or even higher biological activity. The best studied example is equol ${ }^{(15)}$.

Generally polyphenols offer benefits under conditions of stress, which includes oxidative stress and exposure to toxins e.g. due to smoking, detrimental microorganisms, chronic or low-grade inflammation, UV exposure. These lead to, mostly age related, changes of tissue structure and function and to chronic diseases, including cardiovascular disease, diabetes, cancer and Alzheimer's disease. Clinical studies support reduction of risk of cardiovascular disease, by effects mediated through LDL, nitric oxide and endothelial function. Many, but not all, epidemiological studies support these observations ${ }^{(9)}$. There are very few long-term intervention studies of more than 3 months with polyphenols, and there are very limited data on the effects of polyphenols in children. A recent meta-analysis of human intervention studies has shown effects of polyphenol rich foods on flow mediated dilation, blood pressure and LDL cholesterol ${ }^{(2)}$.

Furthermore, genetic predisposition and environmental factors may affect the biological outcome and lead to further complications for establishing dose-effect relationships. For these reasons, large clinical trials linking validated biomarkers of exposure to biomarkers of health benefits with consideration of inter-individual variability are scarce for most polyphenols, but are required. Polyphenols may affect 'suboptimal' biomarkers indicative of a disease risk and thus disease prevention, and so the need for a long term exposure at dietary levels is obvious.

In addition to the above issues, all plant foods contain a large range of bioactive molecules, which may act synergistically or antagonistically. At what point should the whole food rather than individual compounds be studied? On the basis of epidemiological and/or in vivo evidence, in vitro assays (cell culture, reporter assays, etc.) using extracts purified individual compounds, AND their potential metabolites could give a first insight into bioactive compounds of a certain food and help to establish and/or select appropriate biomarkers. Once established, there is no substitute for long term human intervention studies consistently applying these biomarkers. In the future, intervention studies could be supplemented by applying genomics, proteomics and metabolomics techniques whenever possible. This requires interdisciplinary, often multicentre approaches, a common registration of clinical trials, such as on clinicaltrials.gov, and the design of future trials to comply with todays well established criteria, including randomization, placebo control, and cross-over. Studies should be designed as far as possible to have potential for inclusion in meta-analyses. Because of the large number of polyphenols, there is a need to focus on and examine the most promising actions of a few selected compounds and end-points. Consideration of realistic effects needs to take into account the dose, since some effects are only seen at 'mega-doses' in vitro. It is essential to focus on certain disease endpoints such as cardiovascular disease via effects on endothelial function, sugar and lipid metabolism, LDL oxidation and anti-inflammatory effects.

\section{Lifespan essential concept}

Unlike vitamins, polyphenols are not required for growth and development and for maintaining vital body functions throughout life. Nevertheless, for selected polyphenols and polyphenol plant sources, there is clinical and epidemiological evidence showing reduction in the risk of chronic diseases. This means that they are, like some other nutrients, essential for reaching the full (genetically-determined) lifespan; we have termed this 'lifespan essential'(1).

\section{Concept of a dietary reference intake}

The Food and Nutrition Board (FNB) of the IOM/NAS is currently expanding the list of nutritional substances that are included in the Dietary Reference Intakes (DRIs) and recognizes that there may be dietary substances other than the classic nutrients for which recommendations should be given. The DRI committee emphasizes that (i) functional endpoints, other than a clinical manifested deficiency, might be important in establishing dietary recommendations and that (ii) biologically active dietary substances, including nutrients, might have substantially different functional outcomes at different intake levels. These functional outcomes could include toxic or other adverse responses, even for nutrients. This takes into account the $\mathrm{U}$ shaped response curve and is based almost entirely on human studies. In fact, for some classical nutrients, the recommended daily allowance (RDA) is close to the upper (recommended) limit (UL) (Fig. 1). The low toxicity of polyphenols would be expected to give rise to a ratio close to that of vitamin $\mathrm{C}$, although data remain to be properly established and correlated. In addition, the exact values will be different for each class of polyphenols and even individual compounds.

The US Department of Agriculture (USDA) and the Department of Health and Human Services are currently updating the government dietary guidelines for 2010 in line with scientific and medical advances. Dietary guidelines have existed since 1980, revisions are made every five years, and they aim to advise the public on how to follow a healthy, balanced diet and indicate to manufacturers the areas of development to boost the healthy profile of their products in line with government guidelines followed by consumer awareness and selection. 


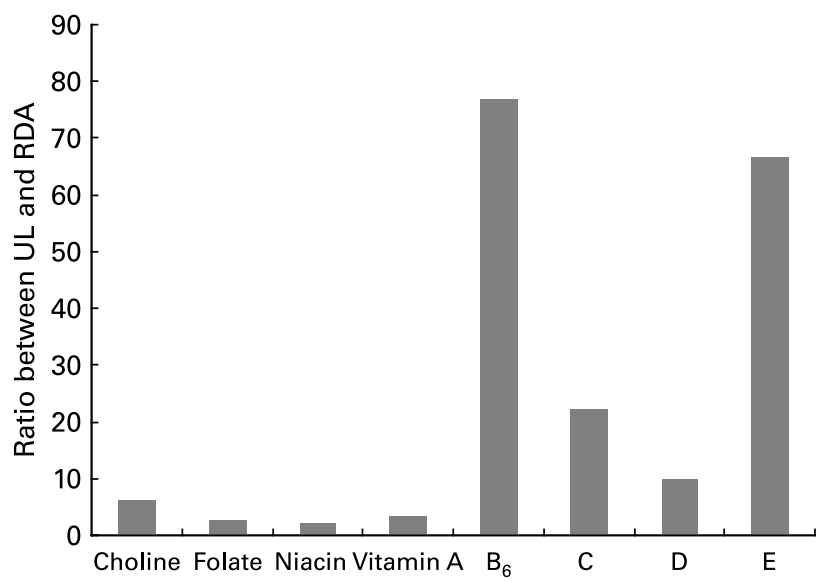

Fig. 1. Ratio between Recommended Daily allowance (RDA) and Upper Limit (UL) values for selected vitamins.

\section{Dietary reference intake for polyphenols?}

The expected intakes of polyphenols from 5-a-day are shown in Table 1, using data derived from the USDA database (http://www.nal.usda.gov/fnic/foodcomp/Data/). Table 1 does not include hydroxycinnamic acids, beverages such as tea or coffee, or cocoa. However, like all nutrients, there is variation with cultivar, year, growing conditions, etc. It can be seen that an individual who consumes 5-a-day could take in $>500 \mathrm{mg}$ of polyphenols daily. Consumption of cocoa, tea or coffee, and consideration of the related hydroxycinnamic acids, could easily increase this by $500-1000 \mathrm{mg}$. However, processed food may contain different amounts. It should not be assumed that a food containing e.g. 2 apples will contain the same amount of polyphenols as 2 apples eaten raw. This is a challenge for the food industry, and a target intake value would help in this respect as the amount of polyphenols could be indicated on the label as a percentage of that actually present in the original food. However, an assessment of dietary intakes in a defined population is difficult to determine due to the lack of comprehensive food composition data of processed foods. For a single polyphenol in food, content estimation is no more difficult than analytical issues for other nutrients, but there are very few approved and certified methods for determining polyphenols in food, unlike for vitamins and minerals.

Could we use currently available data to arrive at a Target Intake value, based on the estimates represented in Table 1? Potential toxicity could arise from intakes of mega-doses of polyphenols via supplements or too highly fortified foods.
This has not been observed in human intervention studies, but some issues have arises for antioxidant vitamins e.g. the meta-analysis of intake of vitamin E, C and selenium ${ }^{(16,17)}$. Dealing with the multiple types of polyphenols needs to be discussed and considered.

Although we and many others propose that polyphenols are important dietary constituents, insufficient data are currently available to establish DRIs. To obtain the required data is an enormous undertaking, requiring public-governmental funding. We highly encourage coordinated research by funding agencies in the area of polyphenols to address the questions above. It should be emphasized that intervention studies which examine a polyphenol dose-response are quite rare and very definitely required.

Dietary recommendations and regulation for any (functional) food enriched in polyphenols, polyphenol-rich extracts and polyphenol based dietary supplements should limit their consumption to dietary reference levels and avoid mega-doses. DRIs should be partly based on epidemiological data concerning the consumption of major food sources in a representative group of the population. However limiting this approach might appear for industry and functional foods, it still opens a wide area of development opportunities: many bioactive polyphenols occur in food sources that are not palatable for all groups of consumers (e.g. the isoflavones in soy products or the green tea catechins), are unstable during processing and storage, or are lost as by-products e.g. during juicing.

\section{The initiatives by the EU (EURECCA) and the USA (ILSI)}

In June 2005, the Food and Drug Administration (FDA), USA rejected a claim on green tea and (mainly breast and prostate) cancer because of the absence of credible evidence (there are many animal and mechanistic studies, but very little human data), and in May 2006 also rejected a claim on green tea and cardiovascular diseases (evidence was supportive, but not conclusive). There is a need for well-conducted clinical studies in this area before further petitions are made. FDA allowed a claim in 1999 on soya (which contains isoflavones, although not specifically stated as such) on lowering levels of total cholesterol and low-density lipoprotein, but this was based on 27 human studies and clearly illustrates that there are not yet enough studies on polyphenols to get a claim approved.

The European Union-funded project, EURECCA, is attempting to harmonise recommended intake values across Europe, since they all draw upon the same publicly available published data. Consideration is being given to polyphenols and other phytonutrients. This review was requested as a result of this project.

Table 1. Intake of polyphenols $(\mathrm{mg})$ from $100 \mathrm{~g}$ of selected foods according to the USDA database

\begin{tabular}{|c|c|c|c|c|c|c|}
\hline & Flavanones & Flavonols & Flavanols & Procyanidins & Anthocyanins & Total \\
\hline Orange & 44 & 0 & 0 & 0 & 0 & 44 \\
\hline Red onion & 0 & 39 & 0 & 0 & 13 & 52 \\
\hline Blueberries & 0 & 4 & 3 & 325 & 113 & 445 \\
\hline Strawberry & 0 & 2 & 4 & 140 & $?$ & 146 \\
\hline Apple & 0 & 4 & 9 & 128 & $?$ & 141 \\
\hline Total & 44 & 49 & 16 & 593 & 126 & 828 \\
\hline
\end{tabular}


ILSI USA has already published a review on the state of the art in preparation for considering DRI values. The conclusions $^{(9)}$ and the main recommendations are to develop reference standards and reference libraries for commonly consumed flavonoids, develop sufficient data to set a DRI or to allow for public health recommendations for one or more of the subgroups of flavonoids, and to determine whether a nutrient model, such as the DRI, is appropriate for bioactive components such as flavonoids. This includes, but is not limited to, determining the risk of deficiency and/or excess in certain instances (e.g. specific tissues or special populations).

\section{References}

1. Holst B \& Williamson G (2008) Nutrients and phytochemicals: from bioavailability to bioefficacy beyond antioxidants. Curr Opin Biotechnol, 19, 73-82.

2. Hooper L, Kroon PA, Rimm EB, Cohn JS, Harvey I, Le Cornu KA, Ryder JJ, Hall L \& Cassidy A (2008) Flavonoids, flavonoid-rich foods and cardiovascular risk: a meta-analysis of randomized controlled trials. Am J Clin Nutr, in press.

3. Manach C, Scalbert A, Morand C, Remesy C \& Jimenez L (2004) Polyphenols: food sources and bioavailability. Am J Clin Nutr 79, 727-747.

4. Williamson G \& Manach C (2005) Bioavailability and bioefficacy of polyphenols in humans. II. Review of 93 intervention studies. Am J Clin Nutr 81, 243S-255S.

5. Manach C, Williamson G, Morand C, Scalbert A \& Remesy C (2005) Bioavailability and bioefficacy of polyphenols in humans. I. Review of 97 bioavailability studies. Am J Clin Nutr 81, 230S-242S.

6. Kroon PA \& Williamson G (2005) Polyphenols: dietary components with established benefits to health? J Sci Food Agric 85, 1239-1240.
7. Sies H, Schewe T, Heiss C \& Kelm M (2005) Cocoa polyphenols and inflammatory mediators Am J Clin Nutr 81, 304S-312S.

8. Keen CL, Holt RR, Oteiza PI, Fraga CG \& Schmitz HH (2005) Cocoa antioxidants and cardiovascular health. Am J Clin Nutr 81, 298S-303S.

9. Erdman JW Jr, Balentine D, Arab L, Beecher G, Dwyer JT, Folts J, et al. (2007) Flavonoids and Heart Health: proceedings of the ILSI North America Flavonoids Workshop, May 31-June 1, 2005, Washington, DC. J Nutr 137, 718S-737S.

10. Wu X, Beecher GR, Holden JM, Haytowitz DB, Gebhardt SE \& Prior RL (2004) Lipophilic and hydrophilic antioxidant capacities of common foods in the United States. J Agric Food Chem 52, 4026-4037.

11. Kelloff GJ, Crowell JA, Steele VE, Lubet RA, Boone CW, Malone WA, et al. (1999) Progress in cancer chemoprevention. Ann N Y Acad Sci 889, 1-13.

12. Schroeter H, Heiss C, Balzer J, Kleinbongard P, Keen CL, Hollenberg NK, et al. (2006) (-)-Epicatechin mediates beneficial effects of flavanol-rich cocoa on vascular function in humans. Proc Natl Acad Sci U S A 103, 1024-1029.

13. Steffen Y, Schewe T \& Sies H (2007) (-)-Epicatechin elevates nitric oxide in endothelial cells via inhibition of NADPH oxidase. Biochem Biophys Res Commun 359, 828-833.

14. Vita JA (2005) Polyphenols and cardiovascular disease: effects on endothelial and platelet function. Am J Clin Nutr 81, 292S-297S.

15. Setchell KD, Brown NM \& Lydeking-Olsen E (2002) The clinical importance of the metabolite equol-a clue to the effectiveness of soy and its isoflavones. J Nutr 132, 3577-3584.

16. Bjelakovic G, Nikolova D, Simonetti RG \& Gluud C (2004) Antioxidant supplements for prevention of gastrointestinal cancers: a systematic review and meta-analysis. Lancet 364, $1219-1228$.

17. Miller ER III, Pastor-Barriuso R, Dalal D, Riemersma RA, Appel LJ \& Guallar E (2005) Meta-analysis: high-dosage vitamin E supplementation may increase all-cause mortality. Ann Intern Med 142, 37-46 